\title{
Macular-appearing Nodular melanoma Arising from a Preexisting Melanocytic nevus in an Elderly patient--Can "Older Age of Nevus" be Besponsible for Unusual \\ Morphogenesis and Melanomagenesis? \\ B Tas ${ }^{1}$, FA Saydam², M Sagır ${ }^{2}$, A Muhammedoglu $^{3}, \mathrm{U} \mathrm{Izol}^{3}$
}

\begin{abstract}
Malignant melanoma (MM) is the most serious skin tumor whose classical clinical types include lentigo maligna (LM), superficial spreading melanoma (SSM), nodular (NM), and acral lentiginous melanoma (ALM). Clinical appearance of a MM may not always be compatible with its histological type, and sometimes it may express itself with an unusual appearance. We report a 80-year-old man with a NM having a macular appearance, which arose from an acquired melanocytic nevus. The lesion of our patient resembled an SSM clinically, but its histopathological examination proved a NM. Such macular appearance of a NM is an unusual condition. Our case was presented to draw attention to both the misleading macular appearance of the NM, and its unusual nonhierarchical development.
\end{abstract}

Keywords: Malignant melanoma, melanocytic nevus, nodular melanoma, oncogenesis, skin, superficial spreading melanoma

From: ${ }^{1}$ Department of Dermatology, ${ }^{2}$ Aesthetic Plastic and Reconstructive Surgery ${ }^{3}$ Department of Pathology, Bagcilar Research and Training Hospital, Bakirkoy/Istanbul, Turkey.

Correspondence: Dr B Tas, Department of Dermatology, Bagcilar Research and Training Hospital, Atakoy 7-8. Kısım, Martı Sitesi, 14/105, Bakirkoy/Istanbul, Turkey. Fax: +90 2124404000, e mail: betulavc@yahoo.com 


\section{INTRODUCTION}

MM is the most malignant melanocytic tumor, and it is responsible for approximately $95 \%$ of deaths caused by cutaneous cancer (1-3). The lesions are usually located on the trunk in males, while they more frequently occur in the lower extremities in females $(3,4)$. The most common clinicopathological types are SSM and NM (3-5). Rapid clinicopathological progression and short intraepidermal growing phase of a NM are usually unnoticeable, until it becomes nodular in form (6).

\section{CASE REPORT}

An 85-year-old male was admitted to our dermatology clinic complaining of a gradually expanding blemish on his back. According to the patient history, a small, yellowish/light brown melanocytic nevus appeared when he was 50 s, and he noticed some slight changes in color in the lesion, two years previously, but he did not care about these changes. The lesion began to enlarge, and its color changed to irregular patch including darker areas, from 1 year ago.

Dermatological examination showed an irregular bordered, multicolored and $2.5 \times 1.3$ $\mathrm{cm}$ in size pigmented patch which was located on the upper-right side of the back. When palpated, the lesion was smooth-surfaced. The skin type of the patient was Fitzpatrick III. Examinations of rest of the skin and mucous membranes did not show similar lesions or additional pathology. Dermatoscopic examination showed an irregularly bordered and multicolored asymmetric patch which had light-brown, dark-brown and black pigmented areas, irregular distributed globules and dots, a few pseudopods and tongue-like extensions, and small depigmented areas on an irregular reticulated background, surrounded by a fine and assymetric peripheral vascular network (Fig.1). 
There was no regional or systemic lymphadenomegaly or hepatosplenomegaly. Routine laboratory examinations, serum levels of lactate dehydrogenase (LDH), cancer markers and chest X-ray were negative or within the normal limits. The lesion was totally excised with a 3 cm safety margin because the lesion could have been a SSM. The histopathological examination revealed only a few atypical melanocytes in the epidermis, as well as mitotic, multinucleated, hyperchromatic and pleomorphic and epitheloid atypical melanocyte nests which came together as multiple small nodules throughout papillary dermis, in the shape of a big cohesive nodule with an expansive growth pattern. The nest sizes and counts increased toward the reticular dermis, but did not invade it (Fig. 2a). In the lesion, microsatellite, perineural or vascular invasion, and tumor regression were not seen. Tumor-infiltrated lymphocyte score was $(+)$. Growth phase was vertical. Breslow thickness was $3.5 \mathrm{~mm}$, and Clark level was III. Mitotic index was 3/per $\mathrm{mm}^{2}$. Immunohistochemically, the tumor showed immunopositivity with Human Melanoma Black (HMB45) (Fig. 2b) and Melan-A (Fig. 2c). Ki67 proliferation index was 35-40\% (Fig. 2d).

With the histopathological and immunohistochemical findings, the diagnosis of NM was confirmed. After the diagnosis, regional lymph nodes were marked with methylene blue, and sentinal lymph node was excised from the right axilla. Its histopathology did not show any atypical melanocytes, and immunohistochemical staining of the lymph node for Melan-A was negative. No regional or systemic metastases were detected on computed tomography. Based on the evidence, the clinical stage of the lesion was identified as Stage-IIA (pT3aN0M0) according to the 7th edition of the AJCC (American Joint Committee on Cancer) cancer staging manual. Therefore, no additional therapy was implemented to the patient. No recurrence was observed during 22-month follow-up period. 


\section{DİSCUSSİON}

The most common clinicopathological subtype of MM is SSM with $60-70 \%$ incidence, and the second is NM with 10-15\% incidence [4]. MM sometimes may show unusual clinical expressions. A nodular-shaped MM usually expresses a NM. However, the nodular appearance can at times result from the uncommon types of MM such as polypoid or pedunculated melanoma. Such lesions can mimic benign skin lesions including intradermal nevus, fibroepithelial polyp, or pyogenic granuloma $(1,7,8)$. On the other hand, SSM usually begins as a smooth or slightly elevated pigmented spot.

When the vertical growth pattern begins, the lesion turns into a papule followed by nodularity, and sometimes an ulceration develops on the surface (6). Since the clinical appearance of our patient's lesion was macular in appearance, we first thought the lesion could be an SSM. Contrastingly, NM usually starts as a raised, variably pigmented papule that increases in size quite rapidly to become a nodule, and often undergoes ulceration. Therefore, in the development of NM, it is usually considered that an in situ component might be lacking.

When the lesion is first diagnosed, a tumorigenic vertical growth pattern is usually seen due to the short-lived antecedent radial phase. (9-11). In the histopathology of early stage of SSM, junctional activity of the malignant cells is predominant. When the vertical growth phase begins, a few clusters of melanoma cells occur in the dermis (9). Although the clinical appearance of our lesion was similar to an SSM, due to the typical histopathological features of the lesion which were attributed to NM, our lesion was differentiated from an SSM. MM lesions usually occur de nova or arise from a pre-existing dysplastic nevus (12).

In MM development, a "stepwise transformation concept" is the most widely considered theory, which means the tumor develops from a common melanocytic nevus to a dysplastic nevus, and finally, to melanoma in situ, in a sequential process (13). A certain proportion of MMs may arise from large congenital melanocytic nevi. Although the 
development of MM from a benign precursor such as small congenital nevi and acquired nevi is possible, it is believed that the possibility is exceedingly low $(13,14)$.

Annual transformation rate of a single nevus into MM has been reported as low as 1/200,000-300,000 (14). However, Tsao et al. suggested that the individual risk of a nevus transforming into MM increases with the "older age of the nevus" (15). In the process of "nevi senescence", some different mechanisms have been proposed. One of them is "replicative senescence" which is an intrinsic limitation on the number of divisions, while still protecting its metabolic activity $(16,17)$. Another is the "oncogen-induced senescence (OIS)", in which some pro-oncogens such as BRAF, HRAS and NRAS which play crucial roles in melanocyte growth, proliferation and also malignant transformation, can become active. It has been shown that there is a significant and positive correlation between the BRAF activity level above threshold and OIS induction (18).

Additionally, OIS may trigger stress-induced senescence, which also leads to DNA damage and other cellular stresses (19). Therefore, it is thought that BRAF may play an important role in MM development (20). Moreover, activated BRAF gene mutations have been found in up to $82 \%$ of acquired nevi (21). Additionally, UVR exposure leads to an acquired BRAF mutation, but high rates of BRAF mutations have been found in nevi from non-UVRexposed skin, as well (22). Indeed, there are some rare examples of MM development from benign nevi. Lallas et al., reported a case of LM which arose from overlying banal intradermal nevus (14). Tajima et al. reported another case of MM within a preexisting intradermal nevus (23).

In another report, Benisch et al. reported two NM lesions originating from intradermal nevi (24). Similar to the Tsao et al.'s suggestions, these authors speculated that, the reason for this nonhierarghical MM development from an intradermal nevus could be related to advanced age of patients $(14,23,24)$. Our lesion arose from an acquired common melanocytic nevus 
existing for many years. For similar reasons, we thought that the "older age of nevus" might have caused a proliferation-prone imbalance in the levels of pro-oncogens, and sequential triggerings of OIS process and melanomagenesis, in our patient.

The presented case is an unusual example of NM, due to its formation from a preexisting small and acquired common melanocytic nevus, and, its prolonged macular clinical appearance despite the characteristic nodular histology. We do not know yet the cause of such unusual behavior of MM. However, it should be kept in mind that clinical appearance of MM may not always be compatible with its histology, and especially, long-term melanocytic nevi can transform into a melanoma in elderly patients. The exact pathogenesis of this nonhierarghical development of $\mathrm{MM}$ arising from a common nevus, and the presented conflicting clinicopathological features will be better understood in the future, through explaining exact roles of immunoregulatory pathways involved in melanomagenesis, individual antitumor resistance caused by interpatient environmental differences, and, determining which factors play a role in morphogenesis of oncogenic proliferation. 


\section{REFERENCES}

1. Hikawa RS, Kanehisa ES, Enokihara MM, Enokihara MY, Hirata SH. Polypoid melanoma and superficial spreading melanoma different subtypes in the same lesion. An Bras Dermatol 2014; 89:666-8.

2. Belgaumi UI, Shetty P, Shirlal S. Oral malignant melanoma: A case report of an unusual clinical and histologic presentation. Dent Res J (Isfahan) 2013; 10:404-7.

3. Vazquez Vde L, Silva TB, Vieira Mde A, de Oliveira AT, Lisboa MV, de Andrade DA, et al. Melanoma characteristics in Brazil: demographics, treatment, and survival analysis. BMC Res Notes 2015; 8:4.

4. Gamsizkan M, Yilmaz I, Buyukbabani N, Demirkesen C, Demiriz M, Cetin ED, et al. A retrospective multicenter evaluation of cutaneous melanomas in Turkey. Asian Pac $\mathbf{J}$ Cancer Prev 2014;15:10451-6.

5. Erdei E, Torres SM. A new understanding in the epidemiology of melanoma. Expert Rev Anticancer Ther 2010; 10:1811-23.

6. Steiner A, Konrad K, Pehamberger H, Wolff K.Verrucous malignant melanoma. Arch Dermatol 1988; 124:1534-7.

7. Cutler K, Chu P, Levin M, Wallack M, Don PC, Weinberg JM. Pedunculated malignant melanoma. Dermatol Surg 2000; 26:127-9.

8. Megahed M, Hoffmann U, Flür M, Hölzle E. Polypoid malignant melanoma. An aggressive, seldom observed variant of nodular malignant melanoma. Hautarzt 1993; 44:437-9.

9. Mishima Y. Melanocytic and nevocytic malignant melanomas. Cellular and subcellular differentiation. Cancer 1967; 20:632-49.

10. Swetter SM, Ecker PM, Johnson DL, Harvell JD. Primary dermal melanoma: a distinct subtype of melanoma. Arch Dermatol 2004; 140:99-103. 
11. Heenan PJ, Holman CD. Nodular malignant melanoma: a distinct entity or a common end stage? Am J Dermatopathol 1982; 4:477-8.

12. Longo C, Rito C, Beretti F, Cesinaro EM, Piñeiro-Maceira J, Seidenari S, Pellacani G. De novo melanoma and melanoma arising from pre-existing nevus: in vivo morphologic differences as evaluated by confocal microscopy. J Am Acad Dermatol $2011 ; 65: 604-14$.

13. Tucker MA, Halpern A, Holly EA, Hartge P, Elder DE, Sageibel RW. Clinically recognized dysplastic naevi. A central risk factor for cutaneous melanoma. JAMA 1997; 277:1439-44.

14. Lallas A, Zalaudek I, Cota C, Moscarella E, Tiodorovic-Zivkovic D, Catricalà C, et al. Naevus-associated lentigo maligna: coincidence or continuum? Hippokratia 2011; $15: 373-5$.

15. Tsao H, Bevona C, Goggins W, Quinn T. The transformation rate of moles (melanocytic naevi) into cutaneous melanoma: a population-based estimate. Arch Dermatol 2003; 139:282-8.

16. Nathon NF, Lloyd AC. Cell senescence and cancer. Nat Rev Cancer 2001; 1:203-13.

17. Bandyopadhyay D, Timchenko N, Suwa T, Hornsby PJ, Campisi J, Medrano EE. The human melanocyte: a model system to study the complexity of cellular aging and transformation in non-fibroblastic cells. Exp Gerontol 2001; 26:1265-75.

18. Michaloglou D, Vredeveld LC, Soengas MS, Denoyelle C, Kuilman T, van der Horst CM, et al. BRAFE600-associated senescence-like cell cycle arrest of human naevi. Nature 2005; 436:720-4.

19. Campisi J, d'Adda di Fagagna F. Cellular senescence:when bad things happen to good cell. Nat Rev Mol Cell Biol 2006; 8:729-40. 
20. Davies H, Bignell GR, Cox C, Stephens P, Edkins S, Clegg S, et al. Mutations of the BRAF gene in human cancer Nature 2002; 417:949-54.

21. Pollock PM, Harper UL, Hansen KS, Yudt LM, Stark M. High frequency of BRAF mutations in nevi. Nat Genet 2003; 33:19-20.

22. Takata M, Murata H, Saida T. Molecular pathogenesis of malignant melanoma: a different perspective from the studies of melanocytic nevus and acral melanoma. Pigment Cell Melanoma Res 2010; 23:64-71.

23. Tajima Y, Nakajima T, Sugano I, Nagao K, Kondo Y. Malignant melanoma within an intradermal nevus. Am J Dermatopathol 1994; 16:301-6.

24. Benisch B, Peison B, Kannerstein M, Spivack J. Malignant melanoma originating from intradermal nevi. A clinicopathologic entity. Arch Dermatol 1980; 116:696-8. 


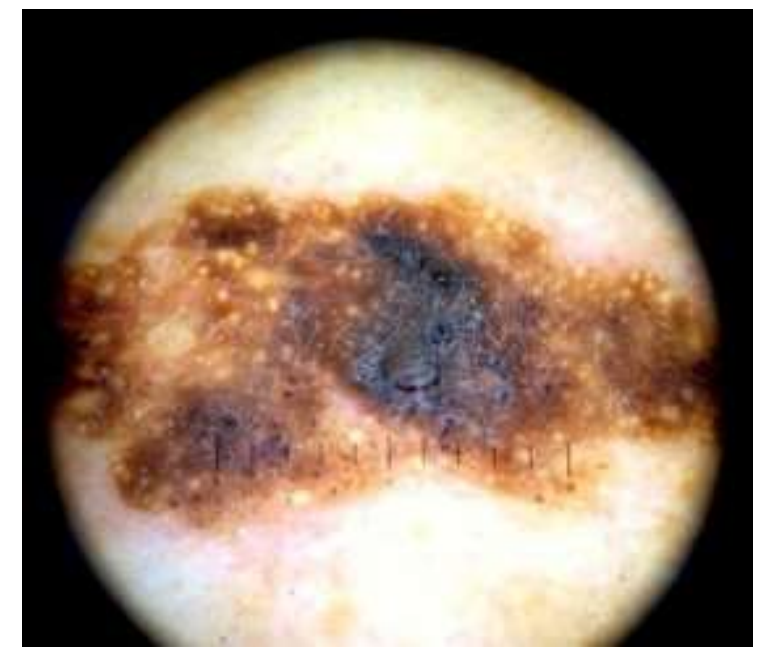

Fig 1: Atypical dermatoscopic appearance of the melanocytic lesion.

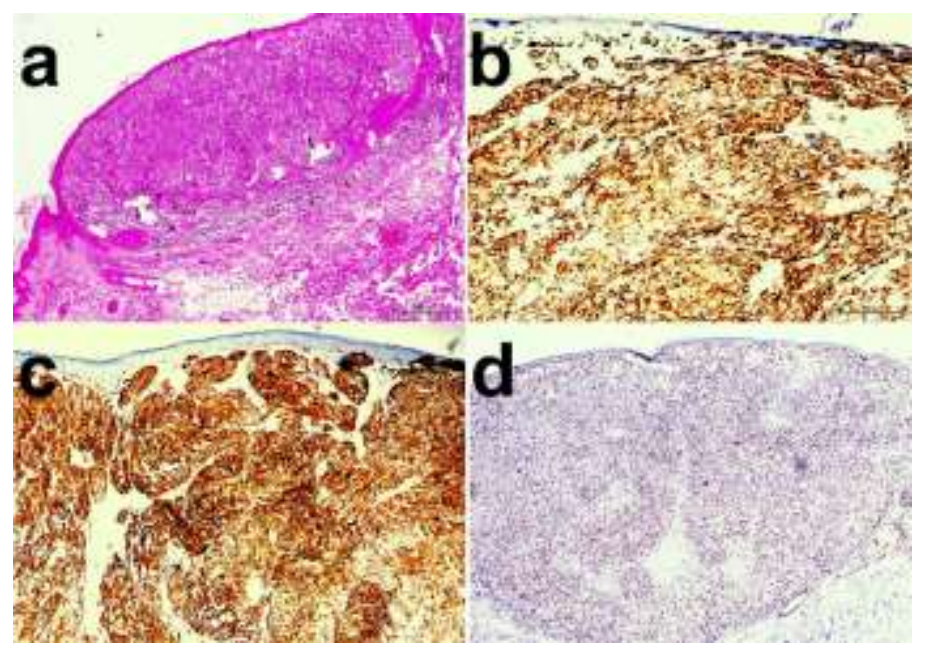

Fig 2: Histological appearance (a. HEX40) and immunohistochemical staining features (b.HMBM45 X100, c. Melan-A X100, d. Ki67 X40) of the nodular patterned dermal tumor. 